\title{
Automated coronal hole identification via multi-thermal intensity segmentation
}

\author{
Tadhg M. Garton*, Peter T. Gallagher and Sophie A. Murray \\ School of Physics, Trinity College Dublin, Dublin 2, Ireland
}

Received 8 June 2017 / Accepted 21 November 2017

\begin{abstract}
Coronal holes $(\mathrm{CH})$ are regions of open magnetic fields that appear as dark areas in the solar corona due to their low density and temperature compared to the surrounding quiet corona. To date, accurate identification and segmentation of CHs has been a difficult task due to their comparable intensity to local quiet Sun regions. Current segmentation methods typically rely on the use of single Extreme Ultra-Violet passband and magnetogram images to extract $\mathrm{CH}$ information. Here, the coronal hole identification via multi-thermal emission recognition algorithm (CHIMERA) is described, which analyses multi-thermal images from the atmospheric image assembly (AIA) onboard the solar dynamics observatory (SDO) to segment coronal hole boundaries by their intensity ratio across three passbands $(171 \AA, 193 \AA$, and $211 \AA)$. The algorithm allows accurate extraction of $\mathrm{CH}$ boundaries and many of their properties, such as area, position, latitudinal and longitudinal width, and magnetic polarity of segmented CHs. From these properties, a clear linear relationship was identified between the duration of geomagnetic storms and coronal hole areas. CHIMERA can therefore form the basis of more accurate forecasting of the start and duration of geomagnetic storms.
\end{abstract}

Keywords: Sun / coronal holes / algorithm / corona / solar wind

\section{Introduction}

The Sun holds a high impact for space weather phenomena through the interactions of solar wind streams with Earth's magnetosphere which can result in geomagnetic storms (Tsurutani et al., 2006). Solar wind streams can damage satellites through differential and bulk charging, and geomagnetic storms may interfere with electrical power networks via geomagnetically induced currents (Boteler, 2001; Huttunen et al., 2008; Marshall et al., 2012; Blake et al., 2016). High speed solar wind streams can be traced back to low density, dark, open magnetic field regions of the corona, known as coronal holes (CHs); (Altschuler et al., 1972; Antonucci et al., 2004; Fujiki et al., 2005; Cranmer, 2009). Here, a new detection algorithm is discussed which segments coronal boundaries from images of the solar corona to ultimately improve predictions of solar wind streams and their properties at $1 \mathrm{AU}$, and hence, their impact on the Earth's magnetosphere.

When observing the corona, $\mathrm{CHs}$ appear as dark regions on the solar disk and off the solar limb (see Fig. 1), particularly in the $193 \AA$ range due to their low density $\left(\sim 4 \times 108 \mathrm{~cm}^{-3}\right)$ and temperatures $(\sim 0.7 \mathrm{MK})$ compared to the surrounding quiet Sun regions $\left(\sim 1.6 \times 109 \mathrm{~cm}^{-3}\right.$ and $\sim 1 \mathrm{MK}$ respectively;

*Corresponding author: gartont@tcd.ie
Phillips, 1995). CHs, while not visible in any other atmospheric solar layer, are indicators of open magnetic field regions which extend far into the heliosphere (Krieger et al., 1973). These extended fields give $\mathrm{CHs}$ an ill-defined cut off height and enable $\mathrm{CHs}$ to be observed extending far out to the outer limits of the low corona. These high altitude $\mathrm{CHs}$ are observed off the solar limb coinciding with near-limb, on-disk CHs, and this phenomena is particularly visible coinciding with polar $\mathrm{CHs}$.

Accurate detection and segmentation of $\mathrm{CHs}$ is made difficult by their irregular profile and comparable intensities to the nearby local quiet Sun regions. Detection becomes further difficult due to off-disk-center $\mathrm{CHs}$ often becoming obscured or occulted by brighter, high-density features such as streamers or active region loops. Initially, $\mathrm{CH}$ segmentation was done by eye based on two-day averages of He I $(10830 \AA)$ images and magnetograms (Harvey \& Recely, 2002) taken by the Kitt peak vacuum telescope. This method adopted the use of magnetograms to separate $\mathrm{CH}$ from other dark coronal features due to the uni-polar nature of $\mathrm{CHs}$ and is still popularly used (Cranmer, 2009). Henney \& Harvey (2005) attempted to automate this detection process and, in turn, created the first automated $\mathrm{CH}$ segmentation algorithm. Post-launch of the Solar and Heliospheric observatory (SOHO), new automated segmentation algorithms were developed that used the clarity of CHs in the $195 \AA$ passband for identification 

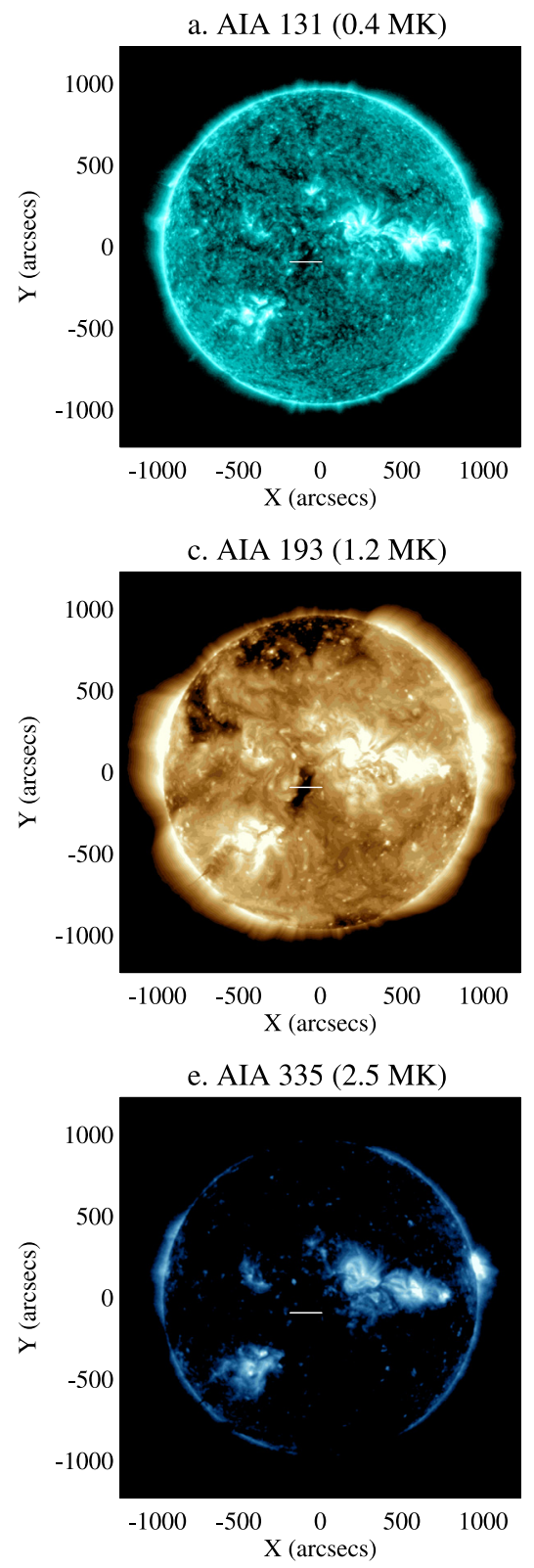
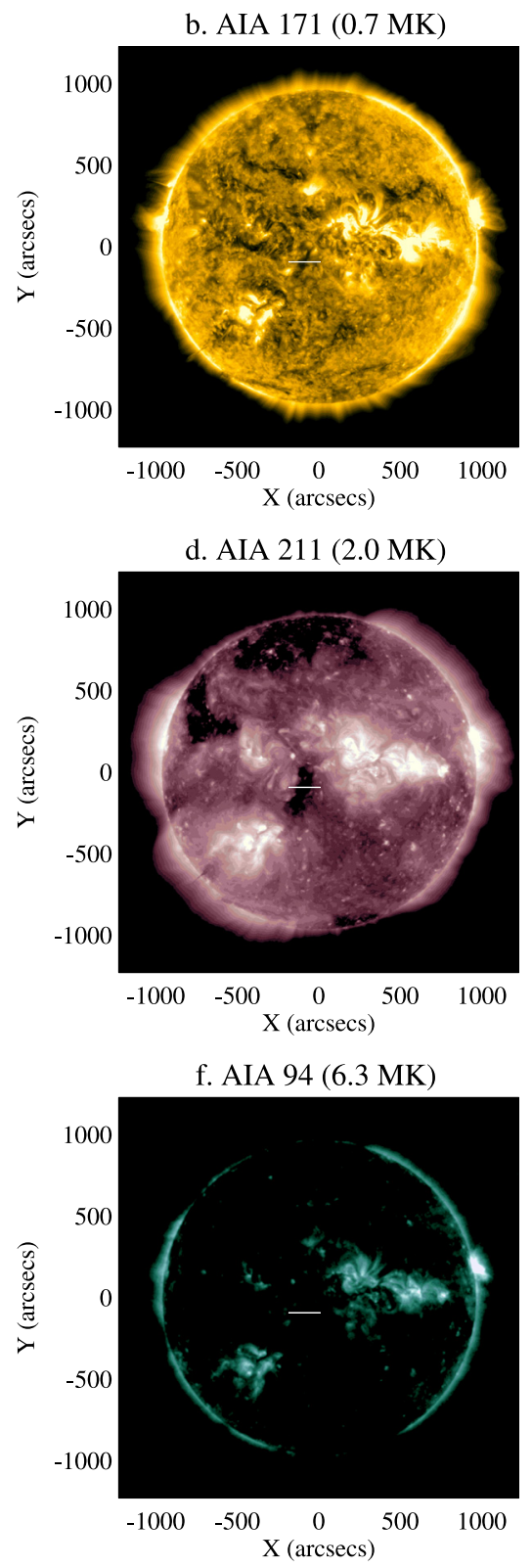

Fig. 1. AIA EUV images in six passbands taken on 22 September 2016 with a $\mathrm{CH}$ located at the central meridian. An intensity cut taken across the $\mathrm{CH}$ is shown in Figure 3.

purposes. Scholl \& Habbal (2008) implemented the primary multi-passband detection method. Contrast enhanced images in the $171 \AA, 195 \AA$, and $304 \AA$ passbands from the extreme ultraviolet telescope on-board SOHO were used to differentiate $\mathrm{CH}$ "candidate features" from quiet Sun and active regions. These candidate features were then separated into $\mathrm{CH}$ s and filaments by comparison with the Harvey \& Recely (2002) He I coronal hole maps and corresponding magnetograms. The coronal hole automated recognition and monitoring algorithm (CHARM), developed by Krista \& Gallagher (2009), uses a histogram-based intensity thresholding technique to differentiate dark coronal regions from quiet Sun regions using only the $195 \AA$ or $193 \AA$ passbands from $\mathrm{SOHO}$ or the solar dynamics observatory (SDO) respectively. These dark coronal candidates were separated into
$\mathrm{CH}$ and non-hole regions through the use of magnetograms. The spatial possibilistic clustering algorithm (SPoCA; Verbeeck et al., 2014) utilizes fuzzy clustering algorithms and distinctions on area and lifetime of features to differentiate $\mathrm{CHs}$ from other features. This algorithm is available for viewing at helioviewer. org. Reiss et al. (2015) designed a machine learning algorithm which uses a global intensity thresholding method and the SPoCA algorithm to separate $\mathrm{CH}$ features from surrounding regions. Recently, Boucheron et al. (2016), developed a detection algorithm which allowed for a varying boundary to search for a maximal change in average intensity between regions inside and outside of this varying contour.

Here, a new method for the detection and segmentation of CHs is discussed. This method is based on multi-thermal 


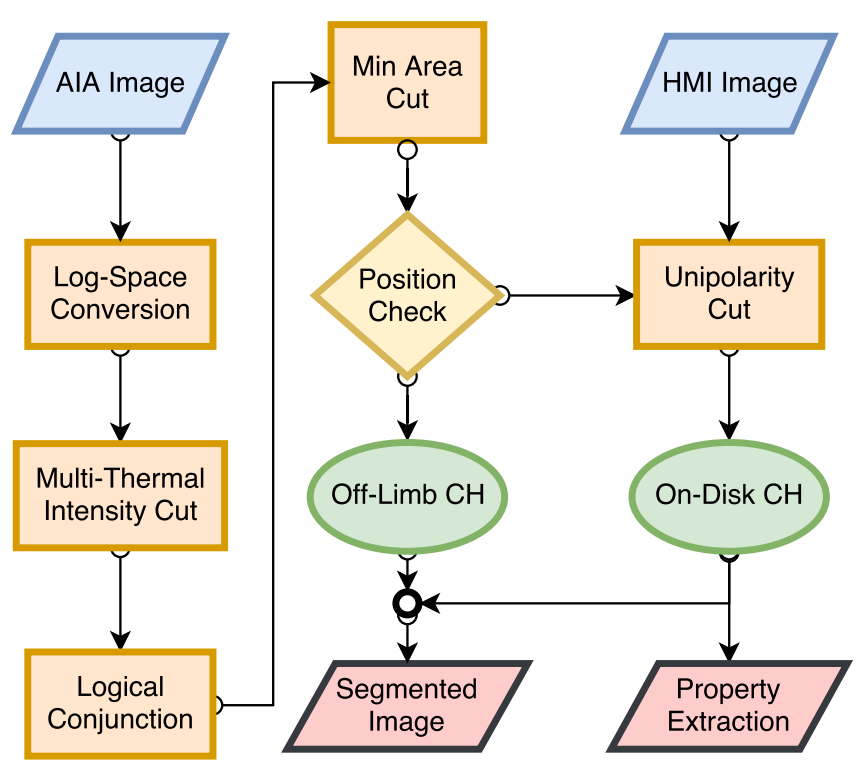

Fig. 2. A flowchart describing the operation of CHIMERA.

intensity segmentation across three EUV wavebands (171 $\AA$, $193 \AA$ and $211 \AA$ ) from the atmospheric imaging assembly (AIA; Lemen et al., 2012) on-board SDO (Pesnell et al., 2012). This method is used in an automated $\mathrm{CH}$ segmentation algorithm, here in named the coronal hole identification via multi-thermal emission recognition algorithm (CHIMERA). CHIMERA analyses intensities in these three wavebands to estimate the temperature and density of individual pixels and then segments pixels with similar properties to a $\mathrm{CH}$. Next, magnetogram analysis removes smaller non- $\mathrm{CH}$ regions that can fall within these constraints by excluding features that do not exhibit the unipolar nature of CHs. In this paper, the stability and accuracy of CHIMERA is demonstrated, and the outputs available for comparison with solar wind measurements at $1 \mathrm{AU}$ are discussed. To categorize a large database of CHs, CHIMERA is automated and runs daily on www.solarmonitor.org/chimera, providing one $\mathrm{CH}$ map per day.

\section{Observations}

CHIMERA was developed for use with image sets from AIA and helioseismic and magnetic imager (HMI) onboard SDO, namely the wavebands: $171 \AA, 193 \AA$, and $211 \AA$ and line of sight magnetograms hmi.M_720s. These EUV passbands were centered on the spectral emission lines of FeIX $(171 \AA)$, FeXII (193 $\AA$ ), and Fe XIV $(211 \AA)$ and cover a peak temperature range of $7 \times 10^{6}-2 \times 10^{7} \mathrm{~K}$. Each passband takes $4096 \times 4096$ pixel images at a cadence of $12 \mathrm{~s}$ for AIA and 12 min for low noise HMI enabling accurate, time precise feature segmentation. Due to the unique characteristic temperature responses and emission across the AIA wavebands of each coronal feature, it is possible to segment an individual feature by the ratios and magnitudes of emission in each waveband relative to each other. Here, the extraction of $\mathrm{CH}$ boundaries from the background coronal features is described by segmenting pixels that exhibit unipolarity, low temperature and low relative density compared to surrounding quiet Sun pixels.

\section{Data analysis}

In this Section, the operation of CHIMERA and the methods used to extract $\mathrm{CH}$ boundaries from the three AIA wavelengths (171 $\AA, 193 \AA$ and $211 \AA)$ and one simultaneous snapshot from the HMI (Schou et al., 2012; Scherrer et al., 2012) is discussed. Figure 2 shows a flowchart describing the segmentation and verification steps of CHIMERA, and is explained further in the following sections: multi-thermal intensity segmentation (Sect. 3.1), verification processes (Sect. 3.2), and off-limb CH detections (Sect. 3.3). CHIMERA ${ }^{1}$ was constructed from procedures found in the SolarSoft library (Freeland \& Handy, 1998) with the interactive data language (IDL) programming language.

\subsection{Multi-thermal intensity segmentation}

Due to the thermal and density properties of coronal features, each feature has a distinct emission spectra. For example, $\mathrm{CHs}$ have a low relative temperature and density, hence they have peak emission in the $171 \AA$ passband and low intensity across other passbands. Figure 3 illustrates an intensity cut across a disk center $\mathrm{CH}$ and the contrast, calculated using the Michelson contrast equation (Eq. (1); Michelson, 1927), between this $\mathrm{CH}$ to the surrounding corona plasma, which is only visually noticeable in the $171 \AA$, $193 \AA$ and $211 \AA$ passbands.

$$
C=\frac{I_{\max }-I_{\min }}{I_{\max }+I_{\min }}
$$

For this equation, $I_{\max }$ is the mean intensity of non- $\mathrm{CH}$ pixels near this $\mathrm{CH}$, and $I_{\min }$ is the mean intensity of $\mathrm{CH}$ pixels within the $\mathrm{CH}$ center. By comparing intensities of individual pixels across these three passbands, it is expected for coronal features to cluster by their distinct emission spectra. Figure $4 \mathrm{a}$, c and e shows three scattergrams comparing the intensities of individual on-disk pixels across these three passbands for a selected date, 31 October 2016. Notably, a high density of pixels, contained within the red boxes in Figure $4 a, c$ and e, exhibit low levels of emission. By taking a close examination of these low intensity pixels, 2D histograms, presented in Figure $4 \mathrm{~b}, \mathrm{~d}$ and $\mathrm{f}$, can be created to demonstrate the clustering of points with specific intensities in the analyzed passbands, with red colours implying a higher density of points, and purple/blue implying lower densities. Two regimes appear to exist in each histogram: a large, hotter cluster exists caused by a large number of quiet Sun pixels typically present on the Sun. Smaller clusters also exist, separated from the quiet Sun clusters by valleys, which have a preferred emission in the $171 \AA$ wavelength. This separation of regimes is as expected from the typical temperature and densities of $\mathrm{CHs}$ and the ambient corona. Finding an optimum fit to the minimum valleys between the two regimes can be used to separate $\mathrm{CH}$ candidates from non- $\mathrm{CH}$ regions. This valley is found for $I_{193} \mathrm{~V}$ $I_{171}$ and $I_{211} \vee I_{171}$ by finding a number of minima in $I_{y}$ for fixed values of $I_{x}$. Due to the irregular shape of the valley in $I_{211} \mathrm{~V}$ $I_{193}$, the minima for this instance are instead calculated along

\footnotetext{
${ }^{1}$ github.com/TCDSolar/CHIMERA
} 
a. AIA 131 (0.4 MK; Contrast=0.15)

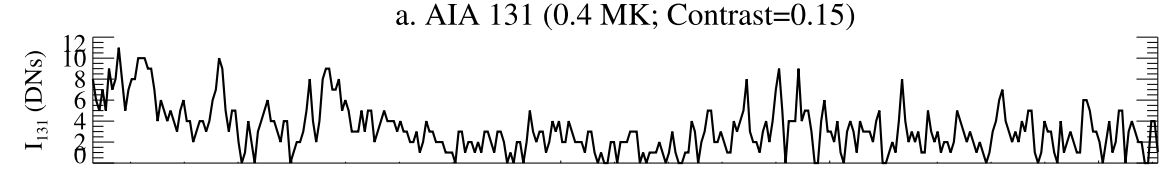

b. AIA $171(0.7 \mathrm{MK}$; Contrast=0.26)

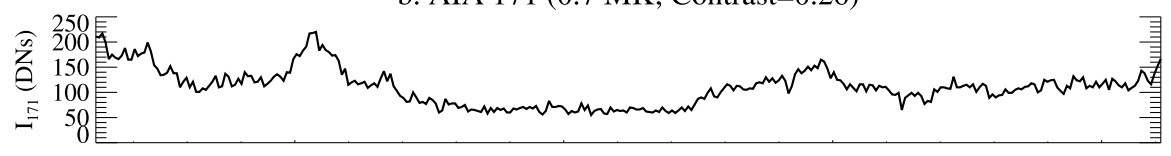

c. AIA 193 (1.2 MK; Contrast=0.59)

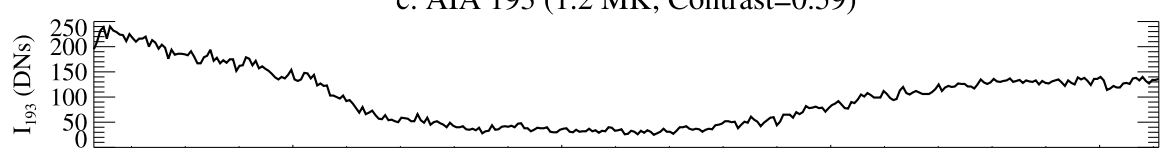

d. AIA $211(2.0 \mathrm{MK}$; Contrast=0.44)

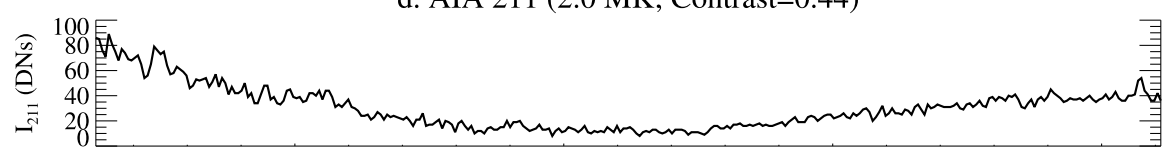

e. AIA 335 (2.5 MK; Contrast=0.11)
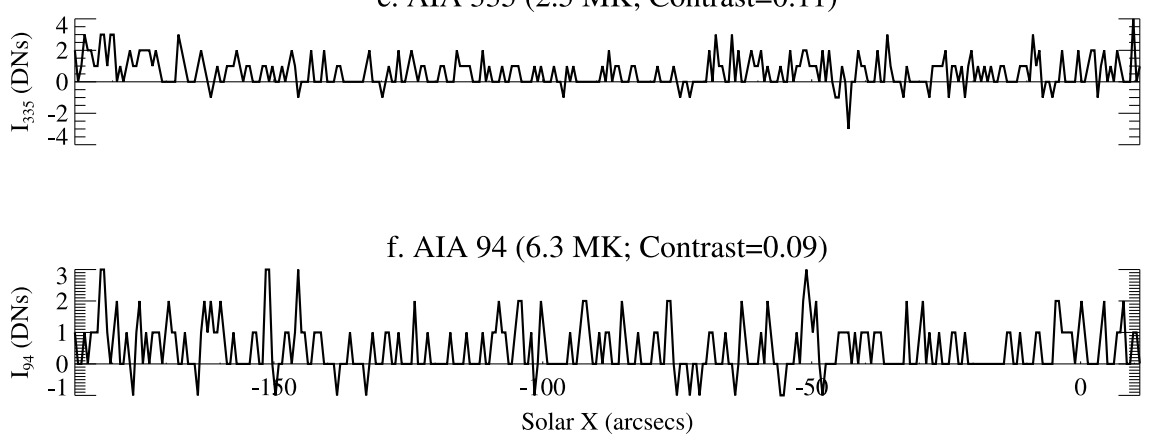

Fig. 3. Intensity cuts across the $\mathrm{CH}$ region at central meridian from Figure 1 on 22 September 2016, in units of digital numbers (DNs), ordered by temperature from top to bottom. An approximation of intensity contrast between the $\mathrm{CH}$ region and the surrounding quiet Sun, calculated using the Michelson contrast equation (1), is displayed in each graph. Difference in intensity between CHs and the surrounding corona is visually noticeable in the $171 \AA$ (b), $193 \AA$ (c), and $211 \AA$ (d) passbands. CHs have a typical temperature of $0.7 \mathrm{MK}$, which means they should produce peak emission in the $171 \AA$ passband.

lines with slopes parallel to the slope of the dominant intensityoccurrence ridge over the entire $I_{211} \mathrm{~V} \quad I_{193}$ regime (implemented here as $\left.\sim I_{211}=(0.3) I_{193}\right)$. Converting these 2D histograms to log-space extends the valleys existing between the clusters, as shown in Figure $5 \mathrm{a}$, c and e. Fitting a line using a least square regression to the minima of the valley in log-space enables the construction of an optimum segmentation line, as follows in equations (2) and (3),

$$
\begin{gathered}
\log I_{y}=m \log I_{x}+\log c \\
=>I_{y}=c I_{x}^{m}
\end{gathered}
$$

where $c=I_{y}\left(I_{x}=0\right), m$ represents the slope of a linear equation, $I_{\mathrm{y}}$ and $I_{x}$ are variable intensity measurements from a solar image. A segmentation line created by this method allows a variation in both slope and shape in intensity-space, as illustrated in Figure 5b, $d$ and $\mathrm{f}$. Feature intensities across these wavebands can vary due to instrument degradation, or may vary with the solar cycle. To accommodate these potential variations in intensity, the segmentation line is altered relative to the average intensity of all on-disk pixels for each wavelength. In the case of low intensity in the $193 \AA$ passband the algorithm alters the line to be more stringent with the $193 \AA$ intensities. This is done by multiplying the line (along the $I_{193}$ axis) by: $\left(\left\langle I_{193}\right\rangle\right) /\left(\left\langle I_{m}\right\rangle\right)$, where $\left\langle I_{m}\right\rangle$ is the measured mean intensity of on-disk pixels for 31 Oct 2016 in the $193 \AA$ passband. This method is similarly performed for the $I_{171}$ and $I_{211}$ measurements. This method gives a simple, rapid, and robust change to the line parameters to continue accurate segmentation of CHs. 

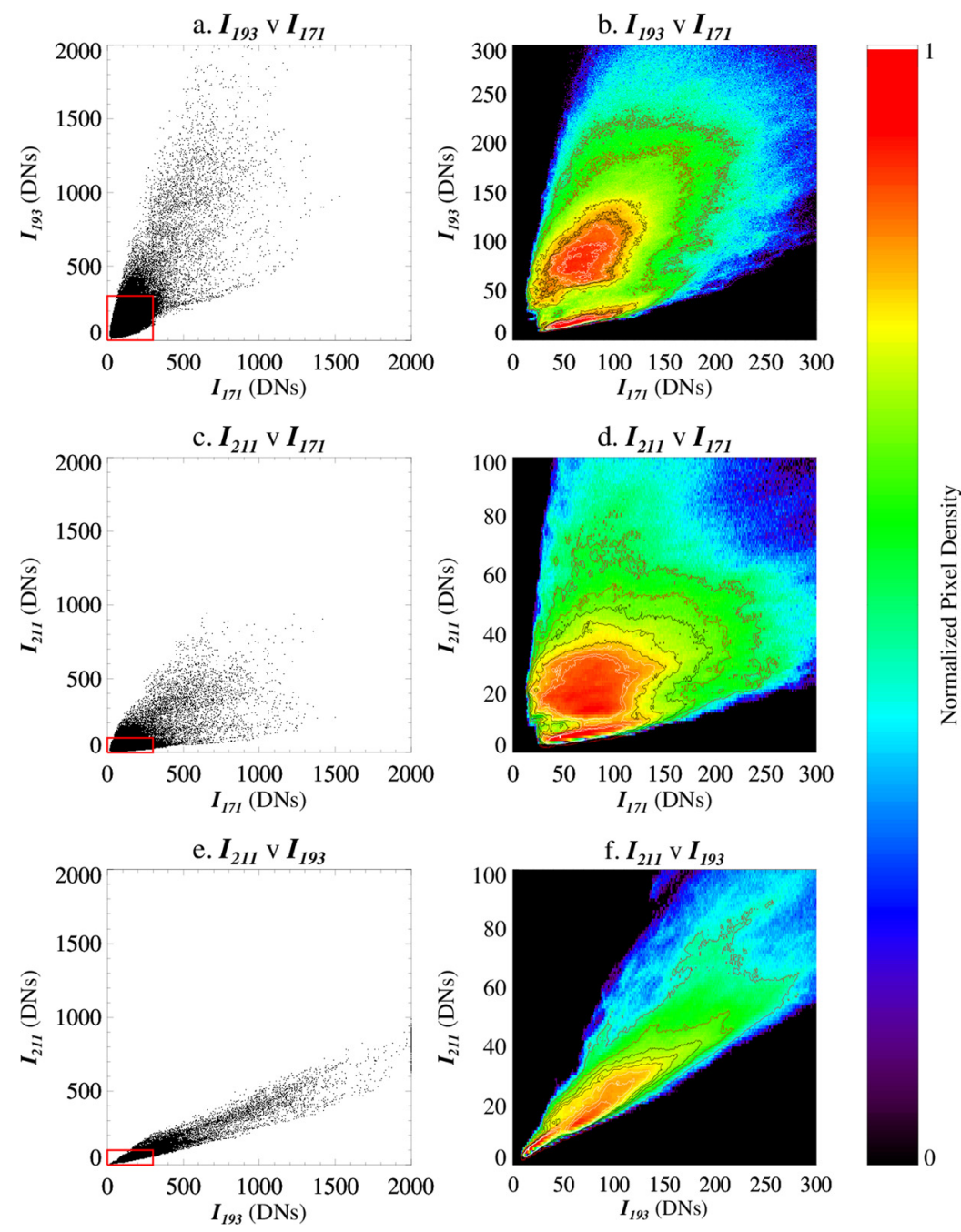

Fig. 4. Intensity of on-disk pixels for 31 October 2016 in $193 \AA$ versus $171 \AA$ (a), $211 \AA$ versus $171 \AA$ (c), and $211 \AA$ versus $193 \AA$ (e), with zoom-ins on lower intensities in (b), (d), and (f), respectively. Colour in these zoom-ins denotes the number of solar disk pixels emitting at these wavelengths, with orange/red implying a large number of pixels and purple/blue implying few.

By taking all regions with intensity ratios below these segmentation curves as $\mathrm{CH}$ candidates, three segmented maps are obtained showing all regions that fit these selection criteria. Figure 6a displays a tri-color AIA image composed of the $171 \AA$ (blue), $193 \AA$ (green) and $211 \AA$ (red) passbands for 31 October 2016, while the three segmented maps shown in Figure $6 \mathrm{~b}, \mathrm{c}$ and $\mathrm{d}$ are created by the three segmentation lines from Figure $5 \mathrm{~b}, \mathrm{~d}$ and $\mathrm{f}$. Notably, these maps have similarly segmented regions, however each is inclusive of additional features not present in the other two segmentations, such as a large filament region being detected in both the $I_{211} \vee I_{193}$ and $I_{193} \vee I_{171}$ segmentations but being absent in the $I_{211} \mathrm{~V} I_{171}$ segmentation. Individually, these maps are insufficient to completely segment $\mathrm{CH}$ regions from other corona features.

\subsection{Verification processes}

As seen in Figure 6b, c and d, each segmented map is excessively inclusive of $\mathrm{CH}$ candidates. However, incorrectly segmented regions are not omni-present in these segmentation maps, while $\mathrm{CH}$ regions are. A logical conjunction of the three segmentations removes these extra features while maintaining the detected $\mathrm{CH}$ pixels. The output binary map of this process is presented in Figure 7, having removed the majority of maldetections while maintaining $\mathrm{CH}$ detections.

From this newly constructed binary map potential erroneous detections are further excluded by rejecting candidates below $1000 \operatorname{arcsec}^{2}\left(\sim 30^{\prime \prime} \times 30^{\prime \prime}\right)$ in size. Small areas could be the result of spurious detections caused by overlapping features or short lived events. This minimum area cut-off follows from the idea of $\mathrm{CHs}$ being expansive regions of open magnetic field. This process removes the majority of erroneous detections, however any remaining non-CH regions are removed through the use of HMI magnetograms and the unipolar nature of CHs. This is completed through an approximation of the mean magnetic field of the $\mathrm{CH}$ candidate. Any candidates not unipolar in nature are removed from detection, i.e. when the mean radial magnetic field strength averaged over the pixels enclosed within the $\mathrm{CH}$ candidates boundary is approximated to zero, $\mathrm{B}_{\mathrm{r}} \approx 0 \mathrm{G}$. The threshold for this cut is calculated relative to the total area of the $\mathrm{CH}$ candidate, with the highest stringency being placed on smaller candidates. A small $\mathrm{CH}$ candidate is accepted if $\mathrm{B}_{\mathrm{r}}$ is 

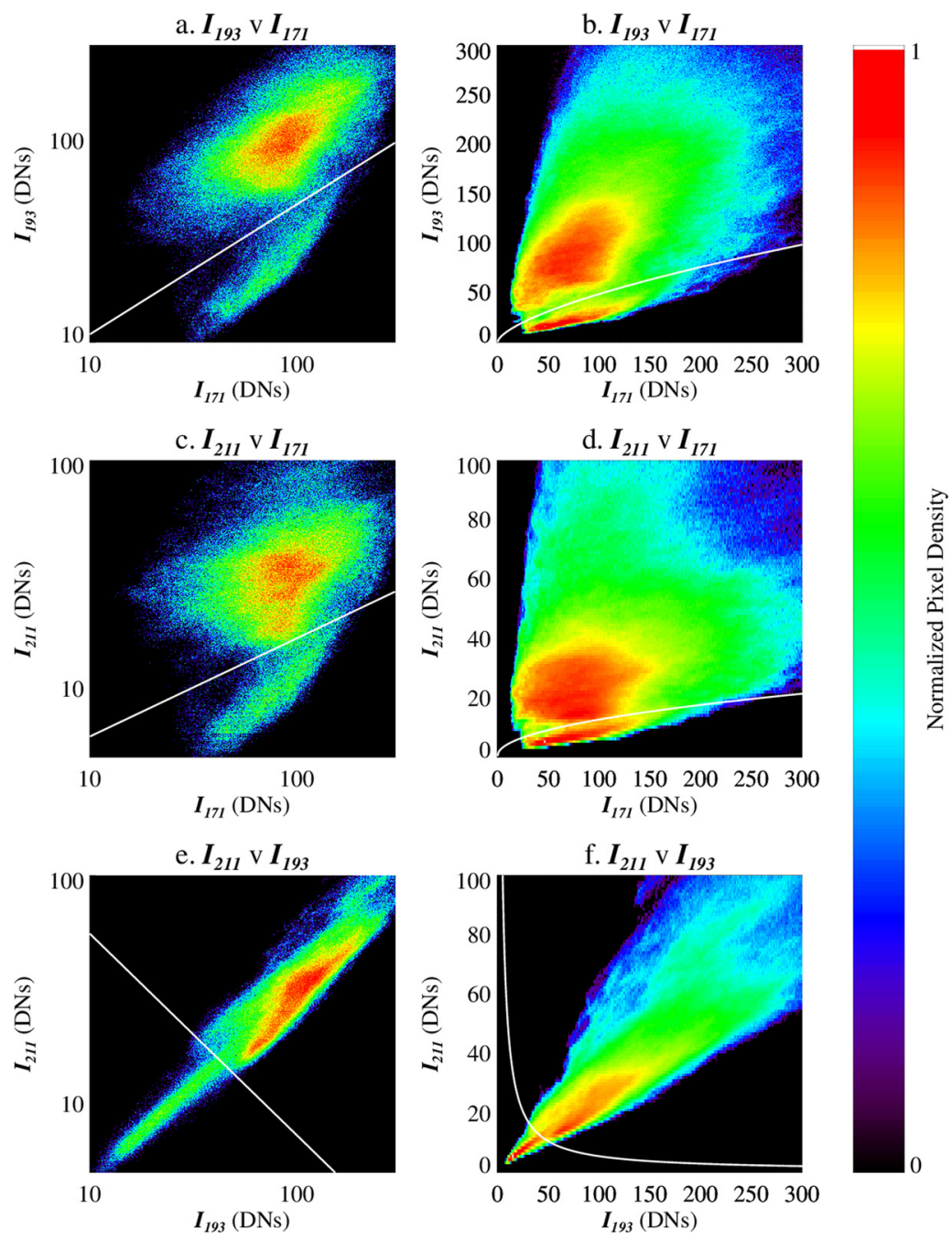

Fig. 5. Optimum intensity segmentation line fits (white) in log space (a) and intensity space (b) of $193 \AA$ versus $171 \AA$, in log space (c) and intensity space (d) of $211 \AA$ versus $171 \AA$, and in log space (e) and intensity space (f) of $211 \AA$ versus $193 \AA$. Segmentation lines in log-space convert to curves in intensity-space, as described by equations (2) and (3).

greater than $1 \mathrm{G}$, and a large $\mathrm{CH}$ candidate with an area above $60000 \operatorname{arcsec}^{2}$ is accepted if $B_{r}$ is greater than $0.1 \mathrm{G}$. This varying stringency prevents large candidates being excluded due to a wider range of polarities being present within their boundaries. These thresholds were found from empirical measurements of magnetic properties of the candidates that were present after the previous conjunction and minimum area cut-off. After these final verification steps, segmented $\mathrm{CH}$ regions are obtained, as displayed in Figure 8.

\subsection{Off-Limb $\mathrm{CH}$ detections}

In CHIMERA's segmentations some detections exist off the solar limb. These high altitude detections are caused by the open magnetic fields that exist within $\mathrm{CH}$ boundaries extending far out into the high corona causing a similar low density and temperature region. These regions, known as plumes, trace out a similar surface shape as that of on-disk $\mathrm{CH}$ regions when projected back onto the coronal surface and have matching thermal properties to that of a $\mathrm{CH}$. Furthermore, these plumes are seen to appear off the polar limbs coinciding with polar $\mathrm{CHs}$ and off the eastern solar limb pre-appearance of an on disk $\mathrm{CH}$. Thus, it is concluded that CHIMERA is capable of detecting the high altitude component of surface CHs. This enables the use of detected regions off the eastern limb as precursors, which can give insight to the appearance, and potential latitudinal width of a $\mathrm{CH}$, before it rotates onto the near side of the solar disk.

\section{Results}

In this section the accuracy, stability and applications of CHIMERA are discussed, as well as the exact properties calculated for every $\mathrm{CH}$, and potential relations that can be observed between CHs and the effects of the solar wind at $1 \mathrm{AU}$.

\subsection{Coronal hole identification}

CHIMERA efficiently and effectively extracts $\mathrm{CH}$ boundaries in a runtime of $\sim 30 \mathrm{~s}$ through the use of the $171 \AA, 193 \AA, 211 \AA$ passbands and HMI magnetograms, and 

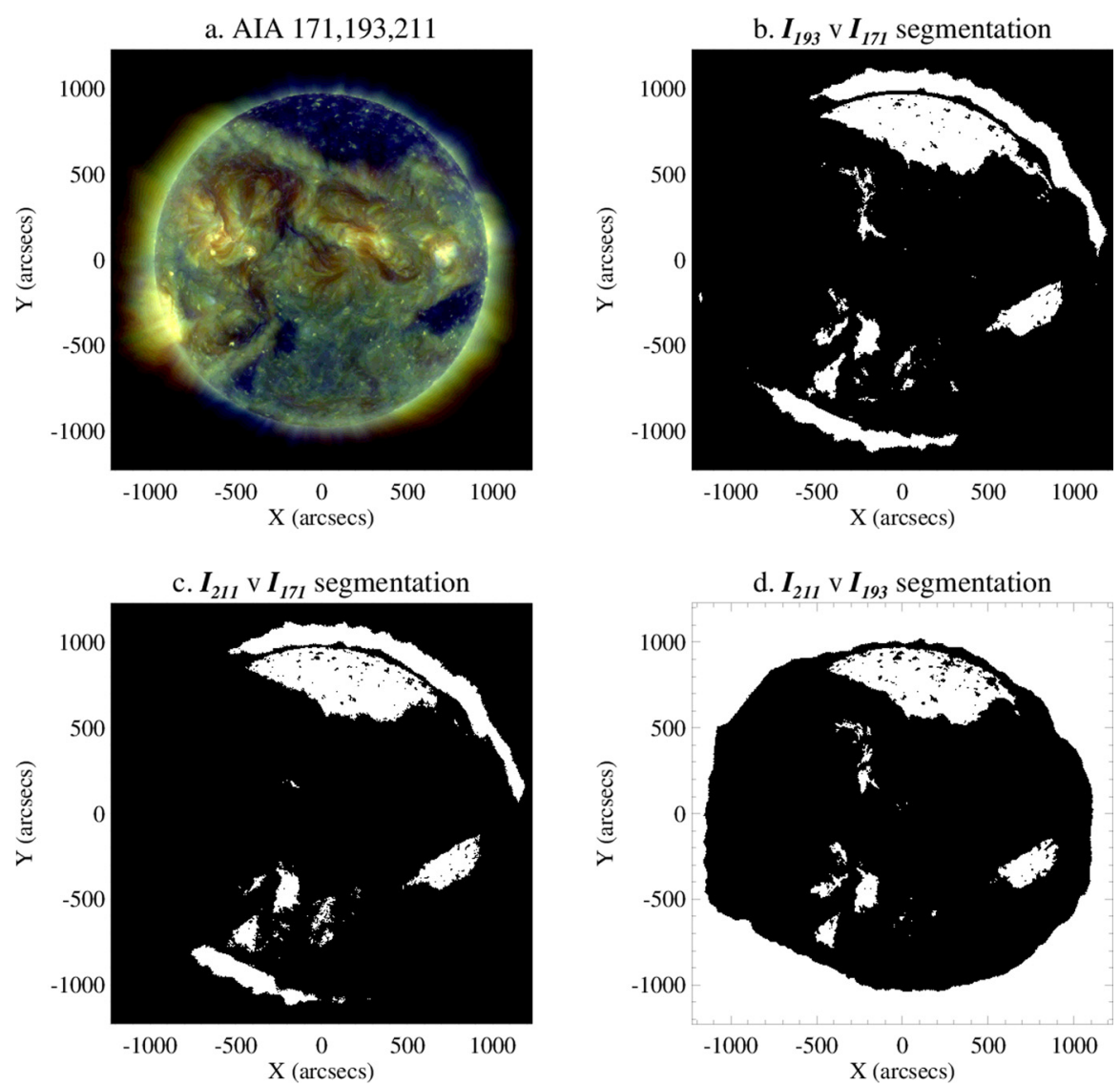

Fig. 6. An example input AIA tri-color image taken on 31 October 2016 in (a), compared to three $\mathrm{CH}$ segmentations obtained from the $I_{211} \mathrm{v} I_{193}$ (b), $I_{193} \vee I_{171}$ (c), and $I_{211} \vee I_{171}$ (d) segmentation curves in Figure $5 \mathrm{~b}, \mathrm{~d}$ and $\mathrm{f}$.
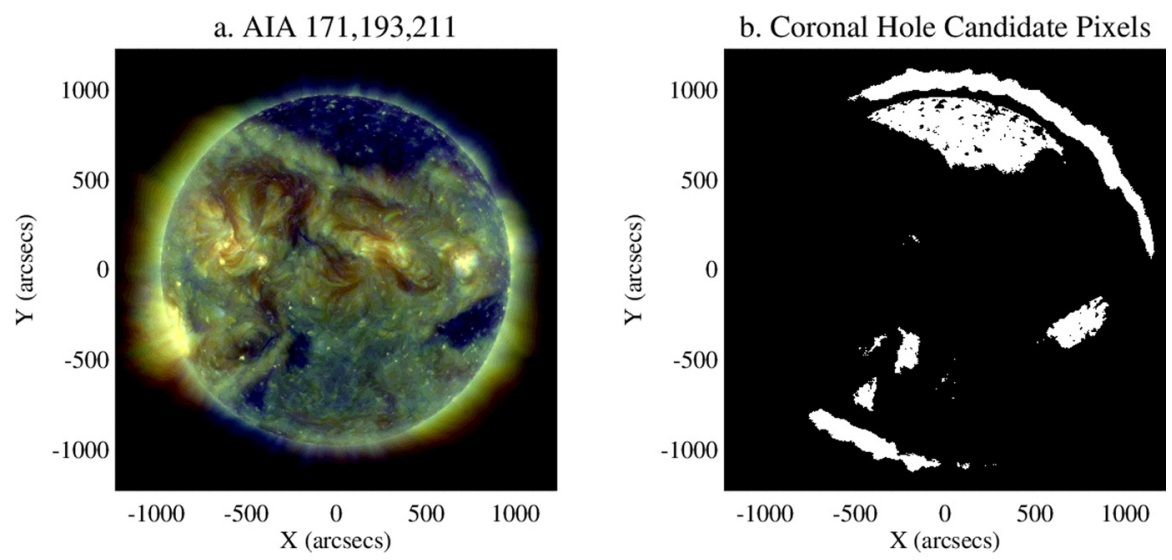

Fig. 7. Through the logical conjunction of the three segmentations in Figure $6 \mathrm{~b}, \mathrm{c}$ and $\mathrm{d}, \mathrm{CH}$ candidates are obtained from CHIMERA that match the thermal properties exhibited by $\mathrm{CH}$ regions on 31 October 2016.

the resulting output is shown in Figure 8. The level of accuracy CHIMERA can attain is demonstrated through a comparison between $\mathrm{CH}$ boundaries extracted by CHIMERA to other extractions methods for 24 October 2016 in Figure 9. This figure displays (a) a manually segmented $\mathrm{CH}$ map from the National Oceanic and Atmospheric Administration (NOAA) Space Weather Prediction Center $(\text { SWPC) })^{2}$, (b) a similar manually segemented map from the Met Office Space Weather ${ }^{2}$ www.ngdc.noaa.gov/stp/space-weather/
Operations Center (MOSWOC) ${ }^{3}$, (c) a segmented map from the automatic solar synoptic analyzer $(\mathrm{ASSA})^{4}$, (d) a segmented map from the $\mathrm{SPoCA}^{5}$, (e) the result of a potential field source surface (PFSS) ${ }^{6}$ extrapolation for comparison of

\footnotetext{
${ }^{3}$ www.metoffice.gov.uk/public/weather/space-weather/

${ }^{4}$ spaceweather.rra.go.kr/models/assa

${ }^{5}$ helioviewer.org

${ }^{6}$ www.lmsal.com/derosa/pfsspack/
} 


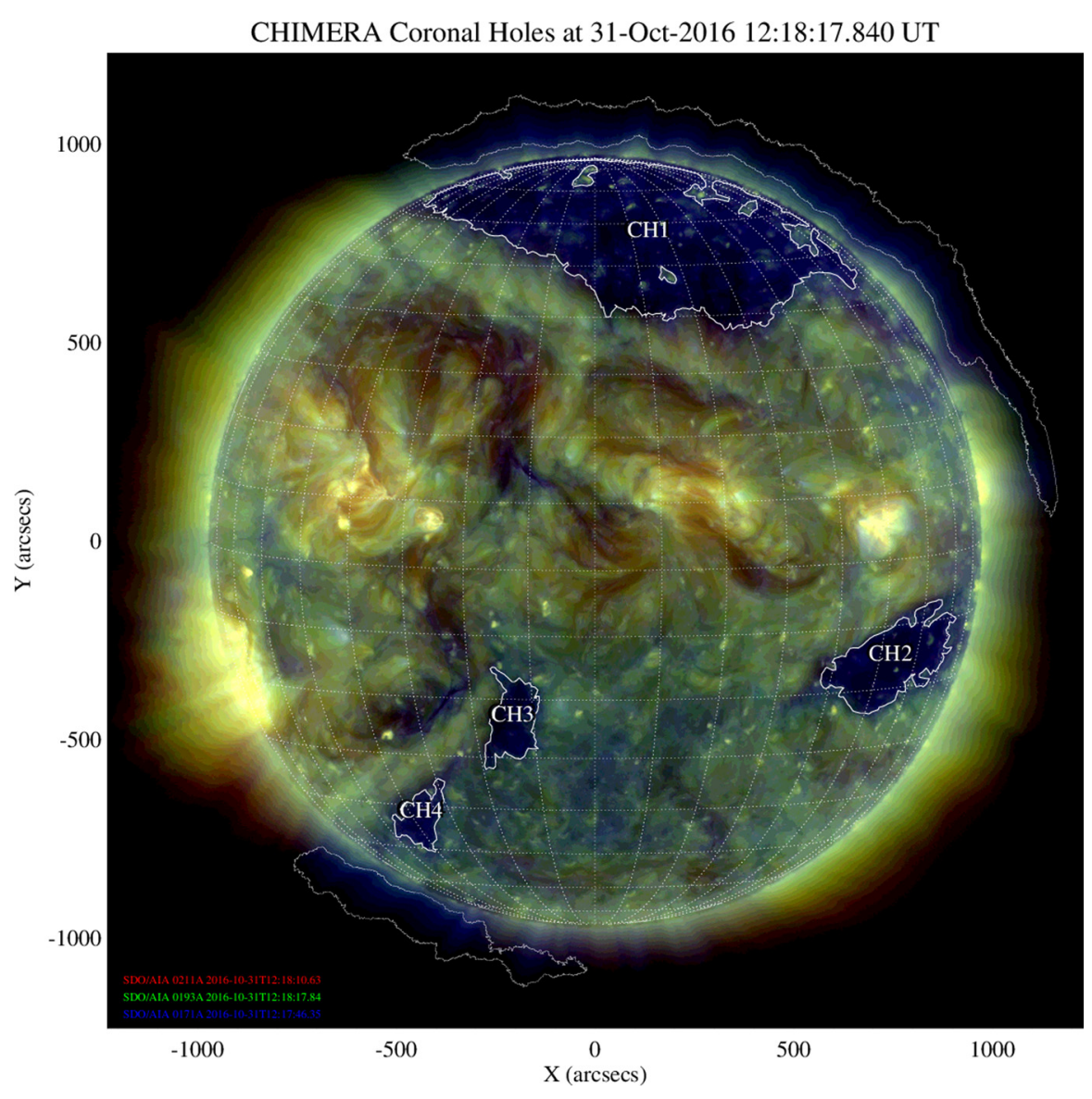

Fig. 8. A segmented image created by CHIMERA for 31 October 2016. CHs regions are extracted from the background quiet Sun and quiet Sun regions trapped within $\mathrm{CH}$ boundaries are ignored and removed from detections. Notably off-limb detections, likely to be plumes, are present in this segmented image due to their high correlation with on-disk $\mathrm{CH}$ regions and matching thermal properties to $\mathrm{CHs}$.

methods with open magnetic field regions, and (f) a segmented map from the CHIMERA ${ }^{7}$ method of segmentation. As can be seen in this comparison, CHIMERAs detailed segmented $\mathrm{CH}$ boundaries follow a similar shape to that of hand-segmented maps, furthermore, CHIMERA visibly better prevents the fracturing of $\mathrm{CH}$ detections than that of the SPoCA algorithm. Due to this high level of correlation of CHIMERA's segmentation ability to that of historically accurate segmentation by eye methods, it is concluded that CHIMERA is capable of accurately segmenting $\mathrm{CH}$ from the quiet Sun. The stability of CHIMERA's detections are demonstrated in Figure 10, where three solar images segmented by CHIMERA for the consecutive days 29-31 January 2017 (a-c) show the shortterm stability of CHIMERA and (d) shows the mid-term stability of CHIMERA by comparing the normalized projected $\mathrm{CH}$ area of six randomly selected $\mathrm{CH}$ regions between January 2016 and July 2017 compared to their centroid longitude. In this plot small scale variations are due to projection effects of the irregularly shape $\mathrm{CH}$ regions. The short-term stability images were selected due to the presence of a large, polar $\mathrm{CH}$ (CH1) passing through the central meridian, which caused a minor storm at $1 \mathrm{AU}$, three days later. This storm registered as a Kp5o storm, according to the World Data Centre for

\footnotetext{
${ }^{7}$ solarmonitor.org
}

Geomagnetism (WDCG), Kyoto ${ }^{8}$. Stability in detections is clearly visible, with each image being a logical one day rotation of the previous with minor boundary morphing expected of a large $\mathrm{CH}$ region over a daily timescale.

\subsection{Coronal hole property extraction}

From these $\mathrm{CH}$ segmentations, $\mathrm{CH}$ properties that may hold the highest impact on solar wind properties at $1 \mathrm{AU}$ can be calculated. Table 1 illustrates all properties extracted from $\mathrm{CH}$ regions by CHIMERA.

Each property extracted by CHIMERA gives some insight into the current and potential geo-effectivity of a $\mathrm{CH}$. $\mathrm{CH}$ area gives possible estimation of the high speed solar wind duration (Krieger et al., 1973; Krista \& Gallagher, 2009) and velocity (Nolte et al., 1976), CH extent and positioning can give further insight into duration, as well as arrival time of solar wind streams (Cranmer, 2002), and magnetic polarity and flux are commonly associated with potential geo-effectivity of solar wind streams. Making these $\mathrm{CH}$ properties readily available outputs of CHIMERA allows a statistical analysis of the detected $\mathrm{CHs}$ and the solar winds they create, an example of which is displayed in Figure 11.

\footnotetext{
${ }^{8}$ wdc.kugi.kyoto-u.ac.jp
} 


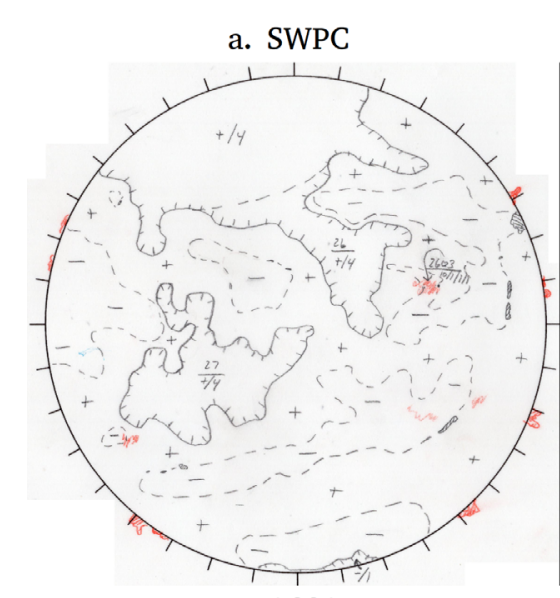

c. ASSA

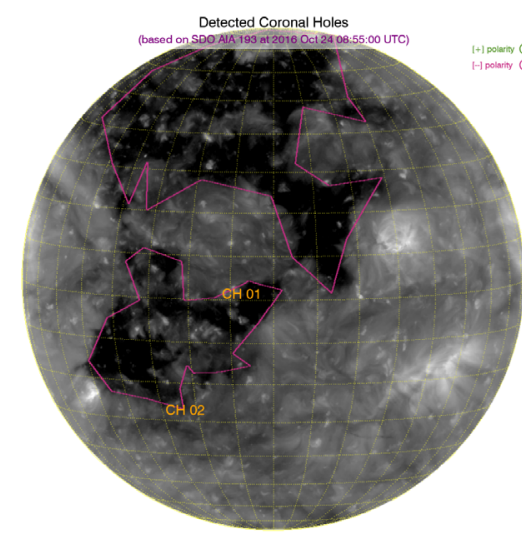

e. PFSS b. MOSWOC

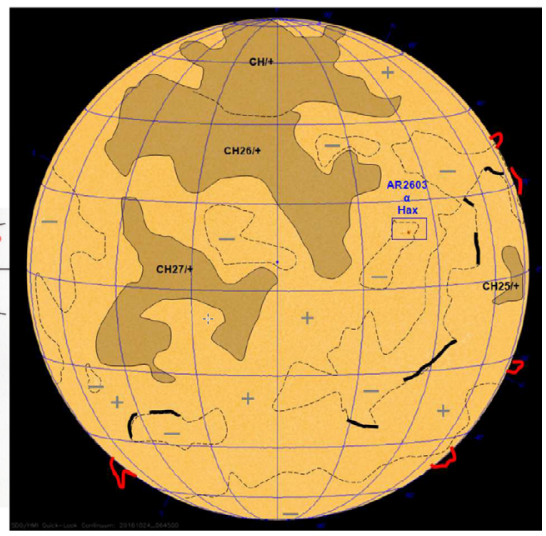

d. SPoCA

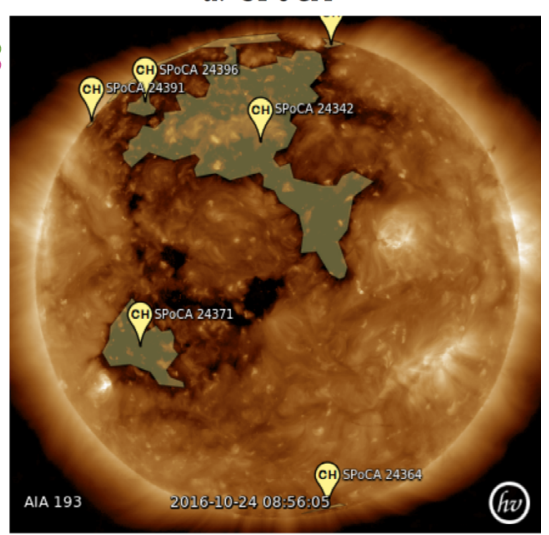

f. CHIMERA

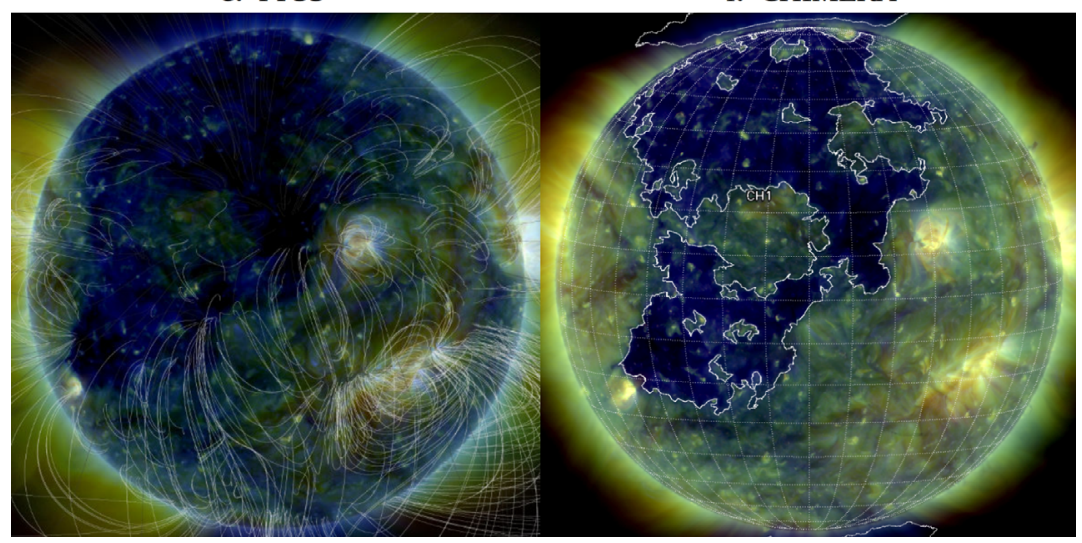

Fig. 9. A comparison of $\mathrm{CH}$ segmentations for 24 October 2016 output by: (a) NOAA SWPC manually drawn maps, (b) MOSWOC manually drawn maps, (c) ASSA segmented maps, (d) SPoCA segmented maps, (e) PFSS models, and (f) CHIMERA segmented maps.

This figure draws a relation between $\mathrm{CH}$ area, geomagnetic storm duration and peak storm intensity, taken from the WDCG. Displayed is a best fit line, $\Delta t=0.26 \pm 0.01 \mathrm{~A}+0.53$ \pm 0.11 , correlating these two properties where $\Delta t$ is the geomagnetic storm duration in days and $A$ is the coronal hole area in percent of the solar disk. From this relationship estimations can be made on the geomagnetic storms a given coronal hole will produce, and, as expected, larger coronal holes will typically produce longer geomagnetic storms.

CHIMERA is however not without limitations, like all automated systems. A predominant weakness in $\mathrm{CH}$ detection algorithms is the inability to detect $\mathrm{CH}$ regions when occulted by brighter, denser regions. Due to pixel intensities being an integration along the line of sight, pixels observing across equal projection of $\mathrm{CH}$ and quiet Sun regions will be seen as quiet Sun by CHIMERA. Normally this phenomenon is of little to no importance, but due to $\mathrm{CHs}$ being a low density feature they are more easily occulted than other coronal features. Furthermore, with solar wind properties having a high correlation with $\mathrm{CH}$ extent and area, inaccurate estimations can cause incorrect estimations of geo-effectivity of CHs.

\section{Conclusions}

A new fast $(\sim 30 \mathrm{~s}$ runtime $)$ and robust $\mathrm{CH}$ identification technique has been developed which uses HMI magnetograms 

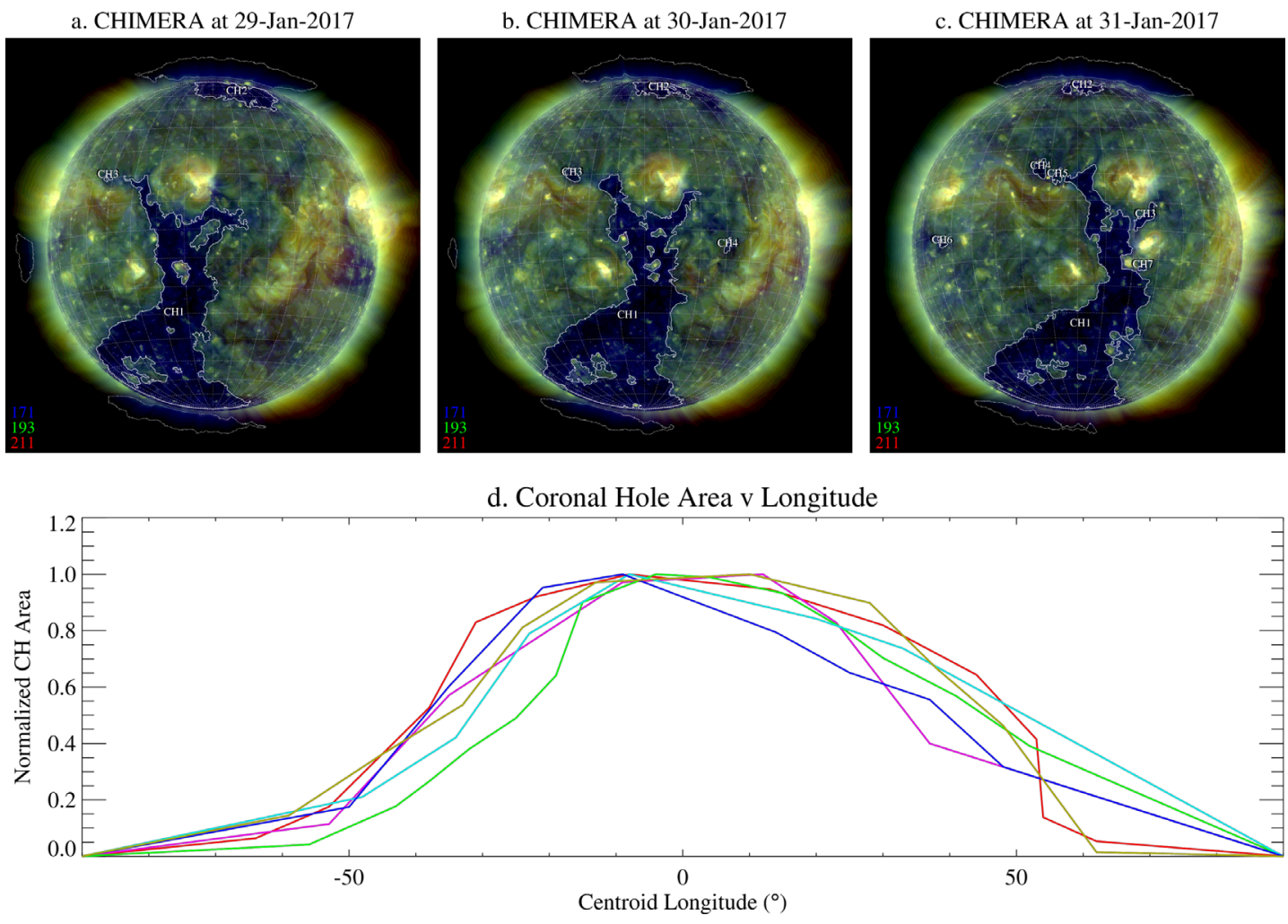

Fig. 10. Three consecutive images taken from 29-31 January 2017 in (a), (b), and (c) demonstrate the short-term stability of CHIMERA's segmentation and tracking abilities. A large geo-effective $\mathrm{CH}(\mathrm{CH} 1)$ is present transversing the central meridian which caused a minor $(\mathrm{Kp} 5 \mathrm{o})$ geomagnetic storm. Mid-term stability is demonstrated in (d), a normalized plot of six randomly selected $\mathrm{CH}$ areas compared to their centroid longitude.

Table 1. $\mathrm{CH}$ properties extracted by CHIMERA.

Extracted property Explanation

$N_{t}$

$X /$ Ycen $_{N}$

$X /$ Yextent $_{N}$

$\Delta \theta$

$A_{\text {tot }, N}$

$A_{\%, N}$

$<B_{\text {los }}>_{N}$

$<B_{\text {los }}^{-/+}>_{N}$

$B_{l o s, N}^{\min / \max }$

$B^{-/+}$

$<\Phi>_{N}$

$<\Phi^{-/+}>_{N}$
Coronal hole identification number for time $t$. This ID number is assigned in descending order of size.

Coronal hole centroid coordinates in arcseconds and Stonyhurst heliographic coordinates.

Most Eastern-Western/Northern-Southern positions in arcseconds.

Longitudinal angular extent of the coronal hole in Stonyhurst heliographic coordinates and absolute degrees.

True coronal hole area. $\left(\Sigma_{p i x} A_{\cos , N}\right)$

Percent coverage of the solar disk by the coronal hole area. $\left(\frac{1 e(+6) \times A_{N}}{\pi R_{s u n}^{2}}\right)$

Mean magnetic polarity along the line of sight. $\left(\frac{\Sigma_{p i x} B_{l o s, N}}{\Sigma_{p i x, N}}\right)$

Mean negative/positive magnetic polarity for a coronal hole along the line of sight. $\left(\frac{\Sigma_{p i x} B_{l o s}^{-/+}}{\Sigma_{p i x, N}^{-l+N}}\right)$

Minimum/maximum magnetic polarity along the line of sight within coronal hole boundaries.

Absolute total polarity for all negative/positive pixels within a coronal hole boundary. $\left(\Sigma_{p i x} B_{\text {los }, N}^{-/+}\right)$

Mean magnetic flux through the surface bounded by the coronal hole boundaries. $\left(\left\langle B_{\text {los }}\right\rangle_{N} A_{\text {tot }, N}\right)$

Mean negative/positive magnetic flux through the surface bounded by the coronal hole boundaries. $\left(\left\langle B_{\text {los }}^{-/+}\right\rangle{ }_{N} A_{\text {tot }, N}\right)$ and the three AIA passbands $171 \AA, 193 \AA$, and $211 \AA$ to segment $\mathrm{CH}$ boundaries. The algorithm classifies every pixel in each image by the exhibited thermal and density properties across these 3 passbands. This unique classification method can be further extended to classify other coronal features such as filaments, which are similarly dark to $\mathrm{CHs}$ but have a peaked emission in the $211 \AA$ passband and are bipolar in nature. Upon completion of segmentation, CHIMERA calculates the centroid, area, latitudinal and longitudinal extent, $\mathrm{CH}$ percentage coverage, and many other properties for comparison with solar wind measurements at $1 \mathrm{AU}$ and predictions of upcoming events up to the following 10-14 days. 


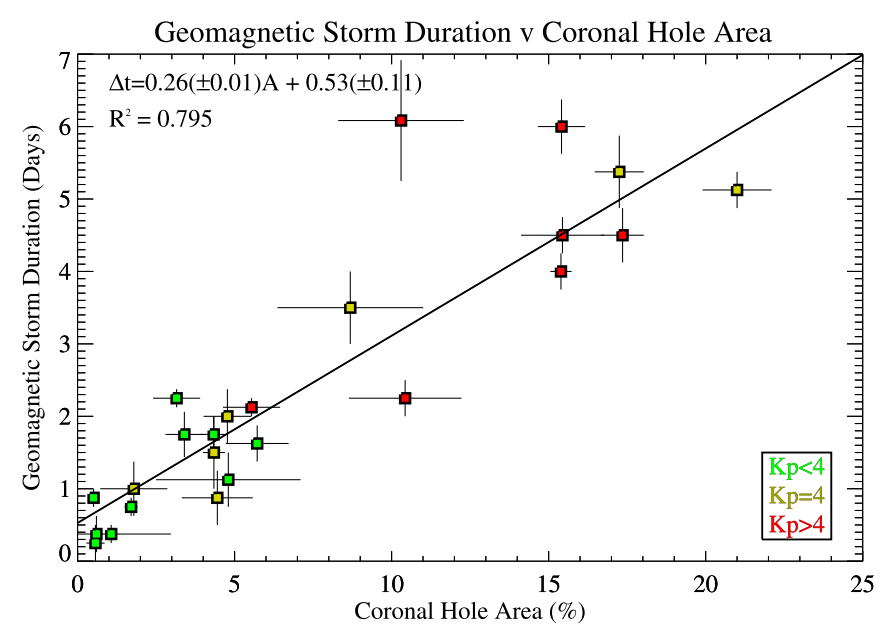

Fig. 11. comparison of measurements of $\mathrm{CH}$ area, the duration of the geomagnetic storms they produced and their peak $\mathrm{Kp}$ index taken from the WDCG. The best fit equation is displayed in the upper left, where $\Delta t$ is the geomagnetic storm duration in days, and $\mathrm{A}$ is the coronal hole area in percent of the solar disk.

The primary limitation of this segmentation method is classification of partially or fully occulted CHs. Low density and dim coronal features are highly susceptible to occultation from brighter features, this is particularly prevalent closer to the solar limb where occultation is more common. This effect implies a $\mathrm{CH}$ can seem to disappear before it reaches the solar limb, which can cause a $\mathrm{CH}$ to become undetectable 1-2 days before expected. These effects can cause errors in boundary segmentation and the calculation of near-limb $\mathrm{CHs}$ properties, however, it is currently impossible to accommodate these effects without the use of multiple viewpoints. Unfortunately, as of yet, there are no multiple viewpoints of the Sun in the three used passbands, although, this method of segmentation could be extended to other passbands which exhibit similar contrasts between $\mathrm{CHs}$ and other coronal features.

CHIMERA can be used to link properties of on-disk $\mathrm{CH}$ regions with the properties of their high speed solar wind streams and the geomagnetic storms they produce. From the $\mathrm{CH}$ properties extracted by CHIMERA and measurements of geomagnetic activity it is possible to draw correlations between these two phenomenon. Figure 11 shows one possible comparison of $\mathrm{CH}$ properties, calculated by CHIMERA, to the properties of the geomagnetic storms they produced. These comparisons can be extended further through machine learning algorithms to draw more relations between $\mathrm{CH}$ properties and the properties of the solar wind streams they produce.

Further study is required on the morphology of $\mathrm{CHs}$ and their interplanetary effects via the high-speed solar wind. Following on from this work, the multi-thermal analysis method of classification can be applied to other coronal features, allowing the segmentation and detection of filament regions or complex active regions and loop structures. Furthermore, outputs of feature properties can be compared statistically with solar wind effects at $1 \mathrm{AU}$ to improve space weather forecasting methods. With the completion of this algorithm, the future aim for this research is to combine this $\mathrm{CH}$ detection software with solar wind models to create a real time emulation of solar CHs and the solar wind they produce up to $\sim 1$ AU. This combined with statistical analyses of solar wind properties will enable accurate predictions of solar wind arrival and lifetimes of geomagnetic storms affecting the Earth's magnetosphere. Furthermore, this method of segmentation will be updated to segment pixels using 3D intensity histograms and tested on GOES-16 X-ray and EUV images in the future.

Acknowledgements. T. M. Garton is supported by a Government of Ireland Studentship from the Irish Research Council (IRC). S. A. Murray is supported by the European Union Seventh Framework Program under grant agreement No. 606692 (HELCATS project) and the European Union Horizon 2020 program under grant agreement No. 640216 (FLARECAST project). The editor thanks Véronique Delouille and an anonymous referee for their assistance in evaluating this paper.

\section{References}

Altschuler MD, Trotter DE, Orrall FQ. 1972. Coronal holes. Sol Phys 26: 354-365, DOI:10.1007/BF00165276.

Antonucci E, Dodero MA, Giordano S, Krishnakumar V, Noci G. 2004. Spectroscopic measurement of the plasma electron density and outflow velocity in a polar coronal hole. $A \& A$ 416: 749-758, DOI:10.1051/0004-6361:20031650.

Blake SP, Gallagher PT, McCauley J, Jones AG, Hogg C, Campanyà J, Beggan CD, Thomson AWP, Kelly GS, Bell D. 2016. Geomagnetically induced currents in the Irish power network during geomagnetic storms. Space Weather 14: 1136-1154, DOI:10.1002/2016SW001534.

Boteler DH. 2001. Space weather effects on power systems. Wash DC Am Geophys Union Geophys Monogr Ser 125: 347-352, DOI:10.1029/GM125p0347.

Boucheron LE, Valluri M, McAteer RTJ. 2016. Segmentation of coronal holes using active contours without edges. Sol Phys 291: 2353-2372, DOI:10.1007/s11207-016-0985-z.

Cranmer SR. 2002. Coronal holes and the high-speed solar wind. Space Sci Rev 101: 229-294, DOI:10.1023/A:1020840004535.

Cranmer SR. 2009. Coronal holes. Living Rev Sol Phys 6: DOI:10.12942/lrsp-2009-3.

Freeland SL, Handy BN. 1998. Data analysis with the solarsoft system. Sol Phys 182: 497-500, DOI:10.1023/A:1005038224881.

Fujiki K, Hirano M, Kojima M, Tokumaru M, Baba D, Yamashita M, Hakamada K. 2005. Relation between solar wind velocity and properties of its source region. Adv Space Res 35: 2185-2188, DOI:10.1016/j.asr.2005.05.057.

Harvey KL, Recely F. 2002. Polar coronal holes during cycles 22 and 23. Sol Phys 211: 31-52, DOI:10.1023/A:1022469023581.

Henney CJ, Harvey JW. 2005. Automated coronal hole detection using He $1083 \mathrm{~nm}$ spectroheliograms and photospheric magnetograms. In K. Sankarasubramanian, M. Penn, and A. Pevtsov, eds., Large-scale Structures and their Role in Solar Activity, vol. 346, Astronomical Society of the Pacific Conference Series, 261 p.

Huttunen KEJ, Kilpua SP, Pulkkinen A, Viljanen A, Tanskanen E. 2008. Solar wind drivers of large geomagnetically induced currents during the solar cycle 23. Space Weather 6: S10002, DOI:10.1029/ 2007SW000374.

Krieger AS, Timothy AF, Roelof EC. 1973. A Coronal hole and its identification as the source of a high velocity solar wind stream. Sol Phys 29: 505-525, DOI:10.1007/BF00150828. 
Krista LD, Gallagher PT. 2009. Automated coronal hole detection using local intensity thresholding techniques. Sol Phys 256: 87100, DOI:10.1007/s11207-009-9357-2.

Lemen JR, Title AM, Akin DJ, Boerner PF, Chou C, et al. 2012. The atmospheric imaging assembly (AIA) on the solar dynamics observatory (SDO). Sol Phys 275: 17-40, DOI:10.1007/ s11207-011-9776-8.

Marshall RA, Dalzell M, Waters CL, Goldthorpe P, Smith EA. 2012. Geomagnetically induced currents in the New Zealand power network. Space Weather 10: S08, DOI:10.1029/2012SW000806.

Michelson AA. 1927. Studies in optics, The University of Chicago Press, Chicago, Ill.

Nolte JT, Krieger AS, Timothy AF, Gold RE, Roelof EC, Vaiana G, Lazarus AJ, Sullivan JD, McIntosh PS. 1976. Coronal holes as sources of solar wind. Sol Phys 46: 303-322, DOI:10.1007/ BF00149859.

Pesnell WD, Thompson BJ, Chamberlin PC. 2012. The solar dynamics observatory (SDO). Sol Phys 275: 3-15, DOI:10.1007/ s11207-011-9841-3.

Phillips K. 1995. Guide to the Sun, Cambridge University Press.

Reiss MA, Hofmeister SJ, De Visscher R, Temmer M, Veronig AM, Delouille V, Mampaey B, Ahammer H. 2015. Improvements on coronal hole detection in SDO/AIA images using supervised classification. J Space Weather Space Clim 5: A23, DOI:10.1051/ swsc/2015025.

Scherrer PH, Schou J, Bush RI, Kosovichev AG, Bogart RS, et al. 2012. The helioseismic and magnetic imager (HMI) investigation for the solar dynamics observatory (SDO). Sol Phys 275: 207-227, DOI:10.1007/s11207-011-9834-2.

Scholl IF, Habbal SR. 2008. Automatic detection and classification of coronal holes and filaments based on EUV and magnetogram observations of the solar disk. Sol Phys 248: 425-439, DOI:10.1007/s11207-007-9075-6.

Schou J, Scherrer PH, Bush RI, Wachter R, Couvidat S, et al. 2012. Design and ground calibration of the helioseismic and magnetic imager (HMI) instrument on the solar dynamics observatory (SDO). Sol Phys 275: 229-259, DOI:10.1007/ s11207-011-9842-2.

Tsurutani BT, Gonzalez WD, Gonzalez ALC, Guarnieri FL, Gopalswamy N, et al. 2006. Corotating solar wind streams and recurrent geomagnetic activity: a review. J Geophys Res: Space Phys 111: A07S01, DOI:10.1029/2005JA011273.

Verbeeck C, Delouille V, Mampaey B, De Visscher R. 2014. The SPoCA-suite: software for extraction, characterization, and tracking of active regions and coronal holes on EUV images. $A \& A$ 561: A29, DOI:10.1051/0004-6361/201321243.

Cite this article as: Garton TM, Gallagher PT, Murray SA. 2018. Automated coronal hole identification via multi-thermal intensity segmentation. J. Space Weather Space Clim. 8: A02 